\title{
Nationwide cohort study of post-gastric bypass hypoglycaemia including 5,040 patients undergoing surgery for obesity in 1986-2006 in Sweden
}

\author{
R. Marsk • E. Jonas • F. Rasmussen • E. Näslund
}

Received: 4 December 2009 /Accepted: 22 April 2010/Published online: 22 May 2010

(C) Springer-Verlag 2010

\begin{abstract}
Aims/hypothesis Symptomatic hypoglycaemia with related confusion, syncope, epilepsy or seizures is a newly recognised complication of gastric bypass surgery for obesity. The incidence of these conditions is not known. We therefore studied the incidence of post-gastric bypass hypoglycaemia and related symptoms in patients who have undergone gastric bypass and a reference cohort from the general population of Sweden.

Methods This is a nationwide cohort study based on national registries with 5,040 persons who underwent gastric bypass, vertical banded gastroplasty or gastric banding for obesity in Sweden between 1 January 1986 and 31 December 2006 and a cohort of ten referents per patient matched for sex and age randomly sampled from the general population. The incidence rates of hospitalisation for hypoglycaemia, confusion, syncope, epilepsy or seizures before and after dates of surgery or inclusion in the reference cohort were studied.

Results Preoperative incidences of hospitalisation for hypoglycaemia were similar in the surgical and referent cohorts. After gastric bypass surgery, the adjusted hazard ratios were significantly elevated for hypoglycaemia (2.7 [95\% CI $1.2-$ $6.3])$, confusion (2.8 [1.3-6.0]), syncope (4.9 [3.4-7.0]), epilepsy (3.0 [2.1-4.3]) and seizures $(7.3[5.0-10.8])$. The proportions of gastric bypass patients and reference
\end{abstract}

R. Marsk $\cdot$ E. Jonas $\cdot$ E. Näslund $(\bowtie)$

Division of Surgery, Department of Clinical Sciences,

Danderyd Hospital, Karolinska Institutet,

SE-182 88 Stockholm, Sweden

e-mail: Erik.Naslund@ki.se

F. Rasmussen

Department of Public Health Sciences, Karolinska Institutet,

Stockholm, Sweden participants affected by hypoglycaemia were very low $(0.2 \%$ and $0.04 \%$, respectively). There was no increased risk of hypoglycaemia after vertical banded gastroplasty or gastric banding compared with the referent population.

Conclusions/interpretation Obese persons who have undergone gastric bypass have an increased risk of hospitalisation for diagnoses associated with post-gastric bypass hypoglycaemia, although few patients are affected.

Keywords Gastric bypass · Hypoglycaemia · Obesity

\section{Introduction}

With increasing rates of obesity the number of antiobesity operations performed is increasing; one of the most common is gastric bypass. Anti-obesity surgery ameliorates diabetes and several other serious comorbidities [1]. One mechanism postulated to improve diabetes after gastric bypass is an augmented incretin response; postprandial plasma concentrations of the potent incretin glucagon-like peptide-1 rise after gastric bypass [2]. Recently hyperinsulinaemia and hypoglycaemia have been recognised as new complications of gastric bypass [3, 4]. Patients have presented with hypoglycaemia associated with confusion, syncope and seizures attributed to changes in glucose homeostasis [5]. Most patients have presented between 2 and 4 years after surgery [5] and in some the condition responds poorly to nutritional therapy, acarbose and somatostatin analogues and may even require partial pancreatectomy $[6,7]$ whereas others have reported that the condition responds to meals that are low in carbohydrates [8].

The incidences of post-gastric bypass severe hypoglycaemia and related symptoms are not known. Our aim was 
to determine the risk of post-gastric bypass hypoglycaemia and associated conditions during a 21-year period compared with a matched cohort taken from the general population in Sweden as reference. In addition, we determined the occurrence of pancreatic surgery after gastric bypass for obesity.

\section{Methods}

Data sources In Sweden all citizens are assigned a unique personal identification number, which allows linkage of medical records nationwide. In this cohort study we used data from the following registries: (1) The Inpatient Care Registry (ICR) from 1 January 1986 to 31 December 2006 provides dates of admissions and International Classification of Diseases (ICD) codes 8, 9 and 10 for diagnoses and surgical procedures (www.who.int/classifications/icd/en/); (2) Statistics Sweden's Multi-Generation Registry includes data on all previous and current residents of Sweden as well as data on emigration; (3) Statistics Sweden's Cause of Death Registry contains data on date and causes of death in the total population of Sweden; and (4) Statistics Sweden's Population and Housing Census includes data on educational level and socioeconomic position. These variables were used to adjust for confounding in the statistical analyses.

Participants The surgical cohort consisted of 5,040 patients $(1,183$ [23\%] men; mean $\pm \mathrm{SD}, 41.0 \pm 10.1$ years of age at surgery) who, according to the ICR, had undergone a primary or secondary (conversion from a restrictive antiobesity procedure; $18.5 \%$ ) Roux-en-Y gastric bypass procedure (GBP), using the procedure codes 4752 , JDF10 or JDF11 with a confirmatory diagnosis of obesity (277.99, 259X, 278A, E66.0, E66.1, E66.8, E66.9). A reference cohort was created by random sampling of ten age-matched and sex-matched people taken from the Multi-Generation Registry for each surgical patient, resulting in 50,400 reference participants $(11,830 \mathrm{men} ; 41.0 \pm 10.1$ years of age at inclusion). The referents were alive at the time when their counterparts in the patient group underwent surgery. Surgical patients and referents were studied with regard to inpatient diagnoses in the ICR from age 18 years to the date of surgery/inclusion, date of death, date of emigration, or to the end of the follow-up study (31 December 2006), whichever came first.

In addition to the main surgical cohort, consisting of all patients treated by gastric bypass procedures for obesity and their referents, we created a subset in which all surgical patients and referents with a diagnosis of diabetes before surgery were excluded. This resulted in 4,027 surgical participants and 40,270 referents.
Finally, we repeated the analyses in 4,366 patients who had undergone vertical banded gastroplasty (VBG; with 43,660 referents) and 2,917 patients who had undergone gastric banding (GB; with 29,170 referents).

Hypoglycaemia and related diseases During the two study periods, before and after surgery/inclusion, the number of hospitalisations for hypoglycaemia (ICD-9: 251; ICD-10: E15-E16) and the surrogates of hypoglycaemia; confusion (ICD-9: 293; ICD-10: F05, R41), syncope (ICD-9: 780C; ICD-10: R55), epilepsy (ICD-9: 345; ICD-10: G40-G41) and seizures (ICD-9: 780D; ICD-10: R568, R252) was also studied in both cohorts, along with the number of hospitalisations for pancreatic surgery $(5,500-5,599$; JLA-JLW) before and after surgery/inclusion. These diagnoses were selected because they have been reported in case series to be associated with post-gastric bypass hypoglycaemia $[4,7$, 8]. Hospitalisations for diabetes mellitus (ICD-8, ICD-9: 250; ICD-10: E10-E14) were studied and the diagnosis was used for adjustments in the Cox proportional hazards regression models.

Ethics and statistics The study was approved by the regional ethics board in Stockholm. Data are presented as number (\%); mean $\pm \mathrm{SD}$, median (range) or incidence rates (per 10,000 person-years) as indicated. Person-time at risk of preoperative morbidity was calculated from 18 years of age to date of diagnosis or date of surgery/inclusion, whichever occurred first. In the analyses of postoperative morbidity, patients were considered to have an event the first time they were diagnosed in the ICR after surgery for hypoglycaemia, confusion, syncope, epilepsy, seizure or pancreatic surgery. Time at risk was calculated from date of surgery/date of inclusion to each of these events, emigration, death or 31 December 2006, whichever came first. In the Cox proportional hazards regression models time of surgery/inclusion was used as the time axis and HRs were adjusted for age at surgery/inclusion, preoperative occurrence of the same diagnosis, socioeconomic position and educational level (model 1). In some models (models 2 and 3) the occurrence of preoperative and postoperative diabetes mellitus was adjusted for. Age at surgery was taken into account as a continuous variable.

\section{Results}

Preoperative incidence rates of inpatient care for hypoglycaemia were similar among patients treated with GBP and referents (Table 1). After surgery, the adjusted HR (model 1, Table 1) was significantly elevated for hypoglycaemia, confusion, syncope, epilepsy and seizures, but not for pancreatic surgery. Adjustment of preoperative diabetes 


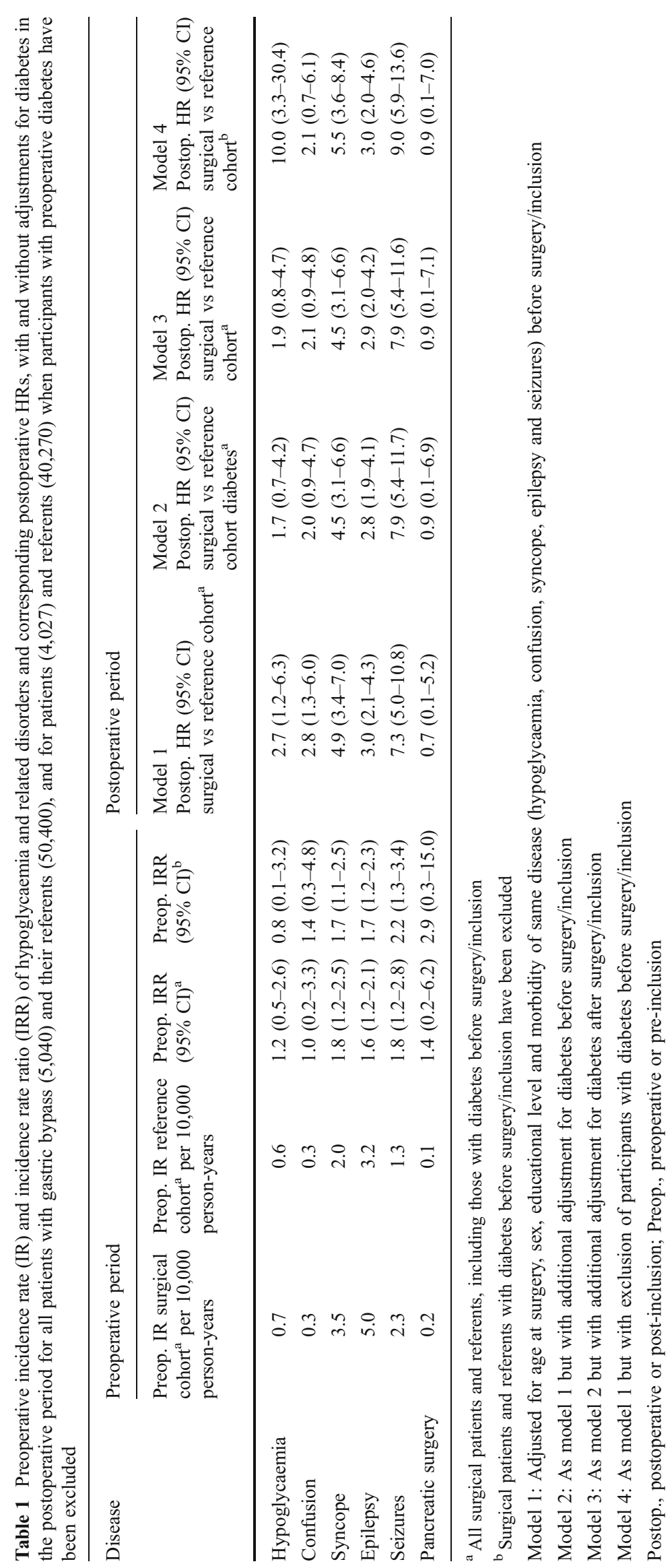


(model 2, Table 1) and additional adjustment for postoperative diabetes (model 3, Table 1) resulted in nonsignificant elevated HRs for hypoglycaemia, confusion and pancreatic surgery, but a significant elevation of the other conditions studied. In the analysis in which preoperative diabetes was excluded (model 4, Table 1), postoperative HR was significantly elevated for hypoglycaemia, syncope, epilepsy and seizures, but not for confusion and pancreatic surgery. The one patient that underwent pancreatic surgery underwent drainage of an abscess. The absolute number of patients affected by any of the studied conditions was low $(\leq 1 \%$; Table 2). The median time from surgery to inpatient care of hypoglycaemia was 2.7 (range 1.0-14.8) years.

The mortality rate (first 3 months after surgery excluded) was greater in the GBP surgical cohort than in the reference cohort (34.5 vs 19.2 per 10,000 person-years). Seven of the $69(10.1 \%)$ deaths in the surgical cohort were accidents and 16 of the $380(4.2 \%)$ deaths in the reference cohort were accounted for as accidents.

In the patients who underwent a restrictive procedure (i.e. VBG or GB) the preoperative incidence rate ratios of hospitalisation for hypoglycaemia compared with the reference population were $1.8(95 \%$ CI $0.5-5.4)$ and 1.5 (95\% CI 0.4-4.3) for VBG and GB, respectively. The postoperative adjusted HRs of hypoglycaemia for VBG and GB compared with the reference population were $1.2(95 \%$ CI $0.6-2.2)$ and 0.9 (95\% CI $0.4-2.1)$, respectively.

\section{Discussion}

Anti-obesity surgery clearly impacts morbidity associated with obesity [1]. However, the surgery is also associated with procedure-related complications. One such complica- tion is hypoglycaemia [8] and this nationwide populationbased study of the occurrence of hypoglycaemia after gastric bypass surgery demonstrates that inpatient care for hypoglycaemia and conditions commonly attributed to hypoglycaemia is significantly more common after surgery than in the general population. However, the actual number of patients affected is low, with less than $1 \%$ of patients affected.

Potential mechanisms include increased insulin secretion because of increased incretin hormones, increased islet functional activity, and increased insulin sensitivity $[4,9]$. The syndrome seems to be procedure-specific and is probably not related to the weight loss per se because there was no significant difference in hypoglycaemia postoperatively in patients that had undergone a restrictive procedure such as VBG or GB compared with the general population. In our study the median time from surgery to symptoms was 2.7 years, which is consistent with the time lag of 1-5 years that has been reported in case studies [5]. Dumping syndrome is seen after gastric bypass surgery $[10,11]$. Many patients with post-gastric bypass hypoglycaemia report dumping early after surgery [5] but this is controlled by dietary intervention, making it unlikely that dumping and post-gastric bypass hypoglycaemia are part of the same disease.

The strength of our study is the nationwide coverage and completeness of Swedish registry data, as well as the fact that all Swedes are covered by a national healthcare system providing affordable access to relatively homogeneous care. Based on our knowledge of Swedish healthcare it is very likely that repeated severe episodes of hypoglycaemia would result in inpatient care. In-hospital registry data are always limited by risks of misclassification and underreporting at the time of discharge from hospital. Further-

Table 2 Number (\%) and risk time in person-years of patients with hypoglycaemia-related conditions (surgical group, $n=5,040$; control group $n=$ 50,400 ) before or after gastric bypass surgery (or inclusion date)

\begin{tabular}{lllll}
\hline & Preop. surgical cohort & Preop. reference cohort & Postop. surgical cohort & Postop. reference cohort \\
\hline Hypoglycaemia, $n(\%)$ & $8(0.16)$ & $64(0.13)$ & $9(0.18)$ & $19(0.04)$ \\
Person-time (years) & 115,674 & $1,156,470$ & 19,430 & 196,608 \\
Confusion, $n$ (\%) & $3(0.06)$ & $29(0.06)$ & $10(0.20)$ & $24(0.05)$ \\
Person-time (years) & 115,701 & $1,156,902$ & 19,450 & 196,586 \\
Syncope, $n$ (\%) & $40(0.80)$ & $227(0.45)$ & $46(0.91)$ & $91(0.18)$ \\
Person-time (years) & 115,462 & $1,155,635$ & 19,322 & 196,321 \\
Epilepsy, $n$ (\%) & $58(1.15)$ & $371(0.74)$ & $40(0.79)$ & $124(0.25)$ \\
Person-time (years) & 115,061 & $1,152,561$ & 19,353 & 196,242 \\
Seizures, $n$ (\%) & $27(0.54)$ & $148(0.29)$ & $50(0.99)$ & $63(0.13)$ \\
Person-time (years) & 115,550 & $1,156,247$ & 19,351 & 196,473 \\
Pancreatic surgery, $n$ (\%) & $2(0.04)$ & $15(0.03)$ & $1(0.02)$ & $12(0.03)$ \\
Person-time (years) & 196,473 & $1,157,013$ & 19,449 & 196,614 \\
\hline
\end{tabular}

Postop., postoperative or post-inclusion; Preop., preoperative or pre-inclusion. 
more, we have no data regarding diabetes treated on an outpatient basis, which also contributes to underestimation of incidence rates. Unfortunately, the ICR does not enable analyses of specific laboratory data during hospitalisation. We are therefore unable to validate the hypoglycaemia diagnosis. Registry data also do not include information on weight development and we could not match surgical patients and the reference population for body mass index. The proportion of patients undergoing gastric bypass surgery has increased over time, whereas the median follow-up time is rather short, which may have resulted in an underestimation of the true incidence of hypoglycaemia because it may not have had time to develop. Hypoglycaemia may result in accidental deaths and as the rate of accidental deaths was higher in the GBP surgical group, this may result in an underestimation of the number of patients with hypoglycaemia.

In summary, this study has demonstrated that there is increased risk of inpatient care for hypoglycaemia following GBP, but not after VBG or GB. Future research should explore and determine the underlying physiological mechanisms. Although incidence rates of hypoglycaemia after GBP surgery appear to be low, our results suggest that both physicians and patients should be aware of this rare but potentially life-threatening complication.

Acknowledgements This study was supported by funds from the Swedish Research Council (medicine), the Stockholm County Council (ALF) and the Karolinska Institutet.

Duality of interest The authors declare that there is no duality of interest associated with this manuscript.

\section{References}

1. Sjöström L, Lindroos AK, Peltonen M et al (2004) Lifestyle, diabetes, and cardiovascular risk factors 10 years after bariatric surgery. N Engl J Med 351:2683-2693

2. Le Roux CW, Welbourn R, Werling M et al (2007) Gut hormones as mediators of appetite and weight loss after Roux-en-Y gastric bypass. Ann Surg 246:780-785

3. Service GJ, Thompson GB, Service FJ, Andrews JC, CollazoClavell ML, Lloyd RV (2005) Hyperinsulinemic hypoglycemia with nesidoblastosis after gastric-bypass surgery. N Engl J Med 252:249-254

4. Patti ME, McMahon C, Mun EC et al (2005) Severe hypoglycemia post-gastric bypass requiring partial pancreatectomy: evidence for inappropriate insulin secretion and pancreatic islet hyperplasia. Diabetologia 48:2236-2240

5. Goldfine AB, Mun EC, Devine E et al (2007) Patients with neuroglycopenia after gastric bypass surgery have exaggerated incretin and insulin secretory responses to a mixed meal. J Clin Endocrinol 92:4678-4685

6. Clancy TE, Moore FD Jr, Zinner MJ (2006) Post-gastric bypass hyperinsulinism with nesidoblastosis: subtotal or total pancreatectomy may be needed to prevent recurrent hypoglycemia. J Gastrointest Surg 10:1116-1119

7. Vella A, Service FJ (2007) Incretin hypersecretion in post-gastric bypass hypoglycemia - primary problem or red herring? J Clin Endocrinol Metab 92:4563-4565

8. Kellogg TA, Bantle JP, Leslie DB et al (2008) Postgastric bypass hyperinsulinemic hypoglycemia syndrome: characterization and response to a modified diet. Surg Obes Relat Dis 4:492-499

9. Meier JJ, Butler AE, Galasso R, Butler PC (2006) Hyperinsulinemic hypoglycemia after gastric bypass surgery is not accompanied by islet hyperplasia or increased beta-cell turnover. Diabetes Care 29:1554-1559

10. Ukleja A (2005) Dumping syndrome: pathophysiology and treatment. Nutr Clin Pract 20:517-525

11. Yamamoto H, Mori T, Tsuchihashi H, Akabori H, Naito H, Tani $\mathrm{T}$ (2005) A possible role of GLP-1 in the pathophysiology of early dumping syndrome. Dig Dis Sci 50:2263-2267 